 \\ www.mdpi.com/journal/mathematics
}

Letter

\section{The Complement of Binary Klein Quadric as a Combinatorial Grassmannian}

\author{
Metod Saniga ${ }^{1,2}$ \\ ${ }^{1}$ Institute for Discrete Mathematics and Geometry, Vienna University of Technology, Wiedner \\ Hauptstraße 8-10, A-1040 Vienna, Austria; E-Mail: metod.saniga@tuwien.ac.at or \\ msaniga@astro.sk; Tel./Fax: +43-1-58801-104363 \\ ${ }^{2}$ Astronomical Institute, Slovak Academy of Sciences, SK-05960 Tatranská Lomnica, Slovak Republic \\ Academic Editor: Palle E.T. Jorgensen
}

Received: 9 May 2015 / Accepted: 5 June 2015 / Published: 8 June 2015

\begin{abstract}
Given a hyperbolic quadric of $\operatorname{PG}(5,2)$, there are 28 points off this quadric and 56 lines skew to it. It is shown that the $\left(28_{6}, 56_{3}\right)$-configuration formed by these points and lines is isomorphic to the combinatorial Grassmannian of type $G_{2}(8)$. It is also pointed out that a set of seven points of $G_{2}(8)$ whose labels share a mark corresponds to a Conwell heptad of PG $(5,2)$. Gradual removal of Conwell heptads from the $\left(28_{6}, 56_{3}\right)$-configuration yields a nested sequence of binomial configurations identical with part of that found to be associated with Cayley-Dickson algebras (arXiv:1405.6888).
\end{abstract}

Keywords: combinatorial Grassmannian; binary Klein quadric; Conwell heptad; three-qubit Pauli group

Let $\mathcal{Q}^{+}(5,2)$ be a hyperbolic quadric in a five-dimensional projective space $\operatorname{PG}(5,2)$. As it is well known (see, e.g., [1,2]), there are 28 points lying off this quadric as well as 56 lines skew (or, external) to it. If the equation of the quadric is taken in a canonical form $\mathcal{Q}_{0}: x_{1} x_{2}+x_{3} x_{4}+x_{5} x_{6}=0$, then the 28 off-quadric points are those listed in Table 1 and the 56 external lines are those given in Table 2. In Table 2, the "+" symbol indicates which point lies on a given line; for example, line 1 consists of points 1,4 and 9. As it is obvious from this table, each line has three points and through each point there are six lines; hence, these points and lines form a $\left(28_{6}, 56_{3}\right)$-configuration.

Next, a combinatorial Grassmannian $G_{k}(|X|)$ (see, e.g., [3,4]), where $k$ is a positive integer and $X$ is a finite set, $|X|=N$, is a point-line incidence structure whose points are all $k$-element subsets of $X$ 
and whose lines are all $(k+1)$-element subsets of $X$, incidence being inclusion. Obviously, $G_{k}(N)$ is a $\left(\left(\begin{array}{c}N \\ k\end{array}\right)_{N-k},\left(\begin{array}{c}N \\ k+1\end{array}\right)_{k+1}\right)$-configuration; hence, $G_{2}(8)$ is another $\left(28_{6}, 56_{3}\right)$-configuration.

It is straightforward to see that the two $\left(28_{6}, 56_{3}\right)$-configurations are, in fact, isomorphic. To this end, one simply employs the bijection between the 28 off-quadric points and the 28 points of $G_{2}(8)$ shown in Table 3 (here, by a slight abuse of notation, $X=\{1,2,3,4,5,6,7,8\}$ ) and verifies step by step that each of the above-listed 56 lines of $\mathrm{PG}(5,2)$ is also a line of $G_{2}(8)$; thus, line 1 of $\mathrm{PG}(5,2)$ corresponds to the line $\{1,4,6\}$ of $G_{2}(8)$, line 2 to the line $\{1,2,4\}$, line 3 to $\{1,3,4\}$, etc.

Table 1. The 28 points lying off the quadric $\mathcal{Q}_{0}$.

\begin{tabular}{ccccccc}
\hline No. & $x_{1}$ & $x_{2}$ & $x_{3}$ & $x_{4}$ & $x_{5}$ & $x_{6}$ \\
\hline 1 & 1 & 1 & 1 & 0 & 0 & 0 \\
2 & 1 & 1 & 0 & 0 & 1 & 0 \\
3 & 1 & 1 & 0 & 0 & 0 & 1 \\
4 & 1 & 1 & 0 & 1 & 0 & 0 \\
5 & 1 & 1 & 1 & 0 & 1 & 0 \\
6 & 1 & 1 & 1 & 0 & 0 & 1 \\
7 & 1 & 1 & 0 & 1 & 1 & 0 \\
8 & 1 & 1 & 0 & 1 & 0 & 1 \\
9 & 0 & 0 & 1 & 1 & 0 & 0 \\
10 & 0 & 0 & 1 & 1 & 1 & 0 \\
11 & 0 & 0 & 1 & 1 & 0 & 1 \\
12 & 0 & 1 & 1 & 1 & 0 & 0 \\
13 & 1 & 0 & 1 & 1 & 1 & 0 \\
14 & 1 & 0 & 1 & 1 & 0 & 1 \\
15 & 0 & 0 & 0 & 0 & 1 & 1 \\
16 & 1 & 0 & 0 & 0 & 1 & 1 \\
17 & 0 & 0 & 1 & 0 & 1 & 1 \\
18 & 0 & 0 & 0 & 1 & 1 & 1 \\
19 & 0 & 1 & 1 & 0 & 1 & 1 \\
20 & 0 & 1 & 0 & 1 & 1 & 1 \\
21 & 1 & 1 & 1 & 1 & 1 & 1 \\
\hline 22 & 1 & 1 & 0 & 0 & 0 & 0 \\
23 & 1 & 0 & 1 & 1 & 0 & 0 \\
24 & 0 & 1 & 1 & 1 & 1 & 0 \\
25 & 0 & 1 & 1 & 1 & 0 & 1 \\
26 & 0 & 1 & 0 & 0 & 1 & 1 \\
27 & 1 & 0 & 1 & 0 & 1 & 1 \\
28 & 1 & 0 & 0 & 1 & 1 & 1 \\
\hline & & & & & &
\end{tabular}


Table 2. The 56 lines having no points in common with the quadric $\mathcal{Q}_{0}$.

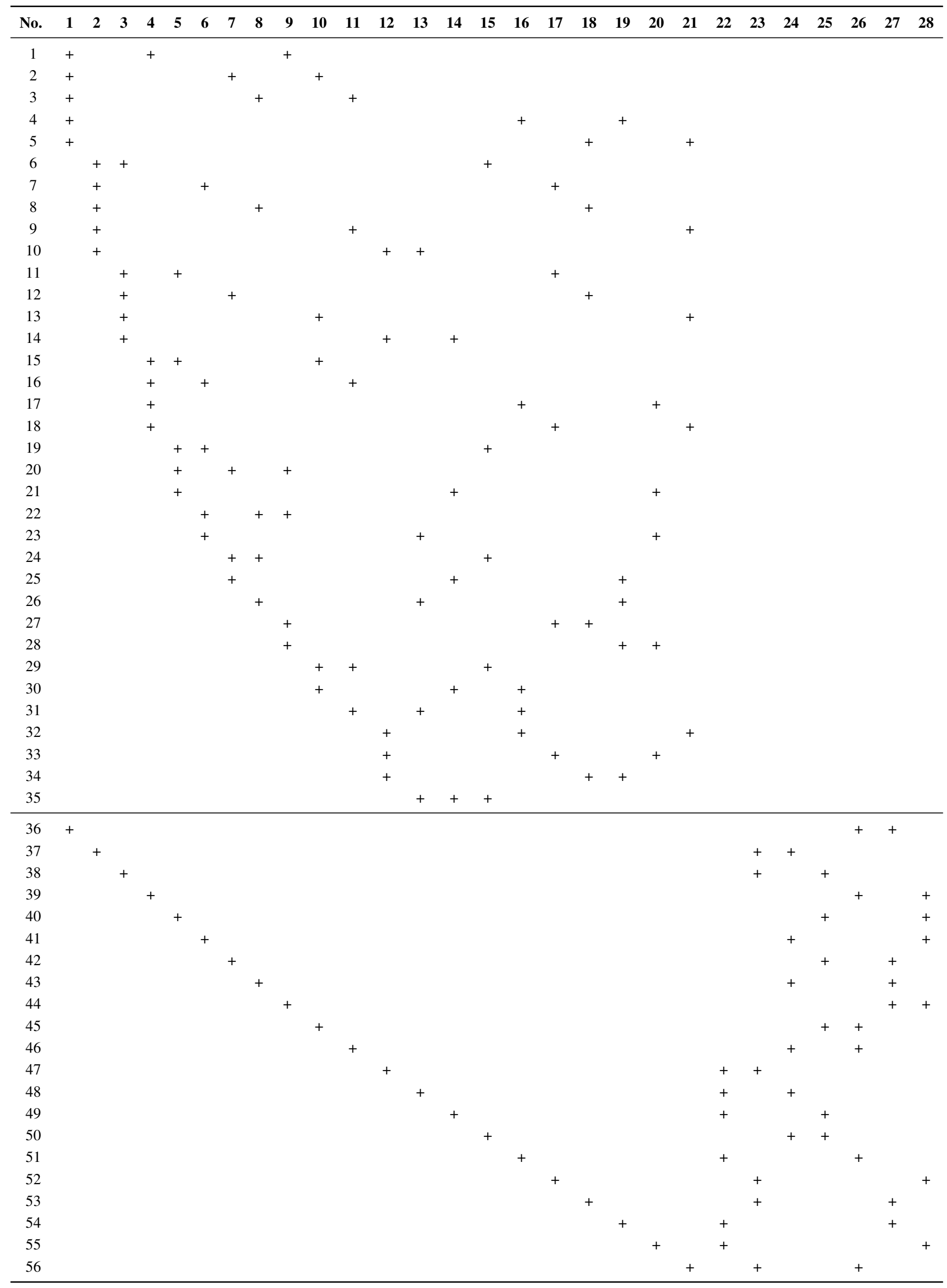


Table 3. A bijection between the 28 off-quadric points and the 28 points of $G_{2}(8)$.

\begin{tabular}{cccc}
\hline off- $\mathcal{Q}_{\mathbf{0}}$ & $\boldsymbol{G}_{\mathbf{2}}(\mathbf{8})$ & off- $\mathcal{Q}_{\mathbf{0}}$ & $\boldsymbol{G}_{\mathbf{2}}(\mathbf{8})$ \\
\hline 1 & $\{1,4\}$ & 15 & $\{2,3\}$ \\
2 & $\{3,5\}$ & 16 & $\{4,7\}$ \\
3 & $\{2,5\}$ & 17 & $\{5,6\}$ \\
4 & $\{4,6\}$ & 18 & $\{1,5\}$ \\
5 & $\{2,6\}$ & 19 & $\{1,7\}$ \\
6 & $\{3,6\}$ & 20 & $\{6,7\}$ \\
7 & $\{1,2\}$ & 21 & $\{4,5\}$ \\
8 & $\{1,3\}$ & 22 & $\{7,8\}$ \\
9 & $\{1,6\}$ & 23 & $\{5,8\}$ \\
10 & $\{2,4\}$ & 24 & $\{3,8\}$ \\
11 & $\{3,4\}$ & 25 & $\{2,8\}$ \\
12 & $\{5,7\}$ & 26 & $\{4,8\}$ \\
13 & $\{3,7\}$ & 27 & $\{1,8\}$ \\
14 & $\{2,7\}$ & 28 & $\{6,8\}$ \\
\hline
\end{tabular}

This isomorphism entails a very interesting property related to so-called Conwell heptads [5]. Given a $\mathcal{Q}^{+}(5,2)$ of $\mathrm{PG}(5,2)$, a Conwell heptad (in the modern language also known as a maximal exterior set of $\mathcal{Q}^{+}(5,2)$, see, e.g., [6] ) is a set of seven off-quadric points such that each line joining two distinct points of the heptad is skew to the $\mathcal{Q}^{+}(5,2)$. There are altogether eight such heptads: any two of them have a unique point in common and each of the 28 points off the quadric is contained in two heptads. The points in Table 1 are arranged in such a way that the last seven of them represent a Conwell heptad, as it is obvious from the bottom part of Table 2. From Table 3 we read off that this particular heptad corresponds to those seven points of $G_{2}(8)$ whose representatives have mark " 8 " in common. Clearly, the remaining seven heptads correspond to those septuples of points of $G_{2}(8)$ that share one of the remaining seven marks each. Finally, we observe that by removing from our off-quadric $\left(28_{6}, 56_{3}\right)$-configuration the seven points of a Conwell heptad and all the 21 lines defined by pairs of them one gets a $\left(21_{5}, 35_{3}\right)$-configuration isomorphic to $G_{2}(7)$; gradual removal of additional heptads and the corresponding lines yields a remarkable nested sequence of configurations displayed in Table 4 . Interestingly enough, this nested sequence of binomial configurations is identical with part of that found to be associated with Cayley-Dickson algebras [7]. Moreover, given the fact that $\mathrm{PG}(5,2)$ is the natural embedding space for the symplectic polar space $W(5,2)$ that geometrizes the structure of the three-qubit Pauli group [8,9], this particular sequence of configurations may lead to further intriguing insights into the physical relevance of this group. 
Table 4. A nested sequence of configurations located in the complement of a hyperbolic quadric of $\mathrm{PG}(5,2)$.

\begin{tabular}{cccc}
\hline \# of Heptads Removed & Configuration & CG & Remark \\
\hline 0 & $\left(28_{6}, 56_{3}\right)$ & $G_{2}(8)$ & \\
1 & $\left(21_{5}, 35_{3}\right)$ & $G_{2}(7)$ & \\
2 & $\left(15_{4}, 20_{3}\right)$ & $G_{2}(6)$ & Cayley-Salmon \\
3 & $\left(10_{3}, 10_{3}\right)$ & $G_{2}(5)$ & Desargues \\
4 & $\left(6_{2}, 4_{3}\right)$ & $G_{2}(4)$ & Pasch \\
5 & $\left(3_{1}, 1_{3}\right)$ & $G_{2}(3)$ & single line \\
6 & $\left(1_{0}, 0_{3}\right)$ & $G_{2}(2)$ & single point \\
7 & & & empty set \\
\hline
\end{tabular}

To conclude this letter, there are a few facts that deserve a special mention. First, the fact that the complement of $\mathcal{Q}^{+}(5,2)$ is isomorphic to the combinatorial Grassmannian $G_{2}(8)$ can be implicitly be traced down even in the original paper of Conwell [5]. As mentioned above, the complement contains eight heptads and each point of the complement can be identified with the (unordered) pair of heptads through it; also the "grassmannian" rule of forming lines on the complement remains valid. After this observation is made, the combinatorial characterization of heptads becomes evident: these are the maximal cliques of the (binary) collinearity. (Clearly, Conwell himself could not formulate his characterization in this combinatorial language.) Second, the fact that removing a complete graph $K_{7}$ from $G_{2}(8)$ one obtains $G_{2}(7)$, and so on, was shown in a more general (" $G_{(n+1)}$ minus $K_{n}$ ") setting in [10] (see also [11]). Finally, it is worth pointing out that the group of automorphisms of the $\left(28_{6}, 56_{3}\right)$-configuration is isomorphic to $S_{8} \cong S L_{4}(2): 2$ (which is the group of collineations and correlations of $\mathrm{PG}(3,2)$, also isomorphic_-via the Klein correspondence-to the group of all collineations of $\mathrm{PG}(5,2)$ preserving a hyperbolic quadric).

\section{Acknowledgments}

This work was partially supported by the VEGA Grant Agency, Project 2/0003/13, as well as by the Austrian Science Fund (Fonds zur Förderung der Wissenschaftlichen Forschung (FWF)), Research Project M1564-N27 "Finite-Geometrical Aspects of Quantum Theory." We thank the anonymous referees for a number of constructive remarks and suggestions.

\section{Conflicts of Interest}

The author declares no conflict of interest.

\section{References}

1. Hirschfeld, J.W.P. Finite Projective Spaces of Three Dimensions; Oxford University Press: Oxford, UK, 1985. 
2. Hirschfeld, J.W.P.; Thas, J.A. General Galois Geometries; Oxford University Press: Oxford, UK, 1991.

3. Prażmowska, M. Multiplied perspectives and generalizations of Desargues configuration. Demonstratio Math. 2006, 39, 887-906.

4. Owsiejczuk, A.; Prażmowska, M. Combinatorial generalizations of generalized quadrangles of order (2,2). Des. Codes Cryptogr. 2009, 53, 45-57.

5. Conwell, G.M. The 3-space PG(3, 2) and its group. Ann. Math. 1910, 11, 60-76.

6. Thas, J.A. Maximal exterior sets of hyperbolic quadrics: The complete classification. J. Combin. Theory Ser. A 1991, 56, 303-308.

7. Saniga, M.; Holweck, F.; Pracna, P. Cayley-Dickson algebras and finite geometry. Discrete Comput. Geom. 2014, arXiv:1405.6888. Available online: http://arxiv.org/abs/1405.6888 (accessed on 3 September 2014).

8. Havlicek, H.; Odehnal, B.; Saniga, M. Factor-group-generated polar spaces and (multi-)qudits. SIGMA 2009, 5, doi:10.3842/SIGMA.2009.096.

9. Thas, K. The geometry of generalized Pauli operators of N-qudit Hilbert space, and an application to MUBs. EPL 2009, 86, doi:10.1209/0295-5075/86/60005.

10. Prażmowska, M.; Prażmowski, K. Binomial partial Steiner triple systems containing complete graphs. 2014, arXiv:1404.4064. Available online: http://arxiv.org/abs/1404.4064 (accessed on 4 June 2015).

11. Petelczyc, K.; Prażmowska, M; Prażmowski, K. Complete classication of the $\left(15_{4} 20_{3}\right)$-congurations with at least three $K_{5}$-graphs. Discret. Math. 2015, 338, 1243-1251.

(c) 2015 by the authors; licensee MDPI, Basel, Switzerland. This article is an open access article distributed under the terms and conditions of the Creative Commons Attribution license (http://creativecommons.org/licenses/by/4.0/). 